\title{
Syringobulbia in pediatric patients with Chiari malformation type I
}

\author{
Arnold H. Menezes, MD, ${ }^{1,2}$ Jeremy D. W. Greenlee, MD, ${ }^{1-3}$ and Brian J. Dlouhy, MD ${ }^{1-4}$ \\ 1Department of Neurosurgery, University of lowa Carver College of Medicine; ${ }^{2}$ Department of Neurosurgery, University of \\ lowa Stead Family Children's Hospital; ${ }^{3}$ Department of Neurosurgery, lowa Neuroscience Institute, University of lowa; and \\ ${ }^{4}$ Department of Neurosurgery, Pappajohn Biomedical Institute, University of lowa, lowa City, lowa
}

OBJECTIVE Syringobulbia (SB) is a rare entity, with few cases associated with Chiari malformation type I (CM-I) in the pediatric population. The authors reviewed all pediatric cases of CM-I-associated SB managed at their institution in order to better understand the presentation, treatment, and surgical outcomes of this condition.

METHODS A prospectively maintained institutional database of craniovertebral junction abnormalities was analyzed to identify all cases of CM-I and SB from the MRI era (i.e., after 1984). The authors recorded presenting symptoms, physical examination findings, radiological findings, surgical treatment strategy, intraoperative findings, and outcomes. SB cases associated with tumors, infections, or type II Chiari malformations were excluded.

RESULTS The authors identified 326 pediatric patients with CM-I who were surgically treated. SB was identified in 13 (4\%) of these 326 patients. Headache and neck pain were noted in all 13 cases. Cranial nerve abnormalities were common: vagus and glossopharyngeal nerve dysfunction was the most frequent observation. Other cranial nerves affected included the trigeminal, abducens, and hypoglossal nerves. Several patients exhibited multiple cranial nerve palsies at presentation. Central sleep apnea was present in 6 patients.

Syringomyelia (SM) was present in all 13 patients. SB involved the medulla in all cases, and extended rostrally into the pons and midbrain in 2 patients; in 1 of these 2 cases the cavity extended further rostrally to the cerebrum (syringocephaly). SB communicated with the fourth ventricle in 7 of the 13 cases.

All 13 patients were treated with posterior fossa decompression with intradural exploration to ensure CSF egress out of the fourth ventricle and through the foramen magnum. The foramen of Magendie was found to be occluded by an arachnoid veil in 9 cases. Follow-up evaluation revealed that SB improved before SM. Cranial nerve palsies regressed in 11 of the 13 patients, and SB improved in all 13.

CONCLUSIONS The incidence of SB in our surgical series of pediatric patients with CM-I was $4 \%$, and all of these patients had accompanying SM. The SB cavity involved the medulla in all cases and was found to communicate with the fourth ventricle in $54 \%$ of cases. Posterior fossa decompression with intradural exploration and duraplasty is an effective treatment for these patients.

https://thejns.org/doi/abs/10.3171/2018.1.PEDS17472

KEYWORDS syrinx; hindbrain herniation; syringomyelia; brainstem; posterior fossa decompression; Chiari

$\mathrm{S}$ YRINGOBULBIA (SB) is a rare entity that presents with a fluid cavity within the brainstem; it is usually slitlike without ependymal lining. 1,20,27,39,40,44 Very few series of Chiari malformation type I (CM-I) with syringomyelia (SM) document the presence of SB. ${ }^{2,59,12,15,19,22,33,40}$
SB may occur with posterior fossa and spinal cord neoplasms, inflammatory disorders such as arachnoiditis and meningitis, and the hindbrain herniation associated with CM-I and Chiari malformation type II (CM-II). ${ }^{3,4,8,24,25,30,38}$ The medulla is most commonly involved in SB, but the

ABBREVIATIONS CM-I = Chiari malformation type I; CM-II = Chiari malformation type II; CN = cranial nerve; $\mathrm{CVJ}$ = craniovertebral junction; $\mathrm{SB}=$ syringobulbia; $\mathrm{SM}=$ syringomyelia. 
cavitation may extend further superiorly, even into the supratentorial compartment. Few case reports focus on SB in the pediatric population with the CM-I. Tubbs et al. retrospectively analyzed MR images from a series of 189 pediatric patients who had been operated on for CM-I and found SB in only 2 of the 189 cases. ${ }^{40}$

We previously reviewed SB in our pediatric population in 2005 and at that time identified 6 patients with SB out of a total of 177 operated on for CM-I between 1984 and 2002 (prevalence 3.3\%). ${ }^{9}$ Given the rarity of SB, here we analyze additional institutional experience in the interest of better understanding the presentation, treatment, and long-term outcomes of SB in pediatric patients with CM-I.

\section{Methods}

Since 1977, a prospective database has been maintained at the University of Iowa Hospitals and Clinics for all abnormalities of the craniovertebral junction (CVJ), including CM-I. This database was searched following institutional research board approval. The criteria for inclusion were 1) diagnosis of SB; 2) diagnosis of CM-I; 3) diagnosis in the MRI era (i.e., after 1984); and 4) age at surgery of 18 years or less. All patients with myelodysplasia and CM-II were excluded, as were all patients with a history of central nervous system neoplasia or infection. We retrospectively reviewed the database and medical records to identify the presenting symptoms, physical examination findings, radiographic abnormalities, intraoperative findings, treatment, and outcomes. Postoperative MRI studies were typically obtained at 6 weeks, 3 months, and 1 and 3 years after the operation. The duration of follow-up ranged from 1 to 20 years.

One patient had previously been described in $1997^{2}$ and was included in the 6 described by Greenlee et al. in 2005 (Cases 1-6 in this report). ${ }^{9}$ A subsequent patient with syringocephaly was reported on in 2015 (Case 10 here). ${ }^{17}$ The current manuscript includes those 7 patients previously reported on in order to extend their follow-up outcomes.

\section{Results}

A total of 326 pediatric patients with CM-I were surgically treated for CM-I between 1984 and 2016, and SB was identified in $13(4 \%)$ of these patients ( 7 female and 6 male). The average age of these 13 patients at the time of surgery was 16 years (range 11-18 years). Four had additional CVJ abnormalities. Table 1 details the presenting symptoms and signs as well as the imaging abnormalities, operative observations, and demographic characteristics for each patient. The postoperative follow-up is also documented.

\section{Presenting Symptoms and Physical Examination Findings}

The most common presenting symptom was headache, which was typically occipital in location. Other common complaints included extremity weakness, diplopia, swallowing difficulties, and snoring/sleep apnea. Sudden onset of diplopia was noted in 2 patients (Table 1). Acute onset of symptoms occurred in 2 patients with violent coughing, and subacute onset was seen in 3 others.
All 13 patients exhibited cranial nerve dysfunction at the time of initial presentation. The most common cranial nerves involved were the glossopharyngeal and vagal nerves (e.g., depressed or absent gag reflex), followed by the trigeminal, abducens, and hypoglossal nerves. Deep tendon reflexes were affected in 9 of 13 patients, and sensory disturbances were present in 7 patients. Horner's syndrome or extremity weakness was found in 3 patients each, as was scoliosis.

\section{Imaging Findings}

MRI at presentation identified the CM-I (with cerebellar tonsillar descent of more than $5 \mathrm{~mm}$ ) in all 13 patients in addition to the SB. All were found to have SM involving the cervical cord. In 9 of the 13 patients, the SM involved the entire spinal cord, and in 1 patient it was cervicothoracic in extent. The SM was limited to the cervical cord in 3 patients.

In all cases, the SB involved the medulla, and in 4 of these the cavity was eccentric to the left side. In only 2 cases did the SB extend superior to the medulla. The SB was felt to communicate with the fourth ventricle in 7 of 13 patients based on MRI.

\section{Surgical Treatment}

All patients were treated with a posterior fossa decompression that included decompression of the foramen magnum and partial (upper) C-1 laminectomy. An intradural procedure was conducted in all cases. A high-resolution intraoperative ultrasound examination was performed in all patients prior to dural opening in an attempt to define the relationship of the SB with the fourth ventricle. During intradural exploration, shrinkage of the cerebellar tonsils and opening of the foramen of Magendie was accomplished. Duraplasty was completed using cervical fascia autograft. Prior to 2004, 3 patients underwent placement of a fourth ventricle to subarachnoid shunt during posterior fossa decompression.

In 4 of 13 cases, the patients had associated CVJ abnormalities, including basilar invagination, proatlas segmentation failure, and atlas assimilation (Table 1). Three of these patients required transoral decompression of the ventral medulla for basilar invagination prior to the posterior fossa procedure; in these cases occipitocervical fusion was also required. One patient had a reducible basilar invagination and was treated with occipitocervical fusion in addition to the posterior fossa decompression procedure.

\section{Operative Findings}

While all 13 patients were found to have fourth ventricle outflow obstruction, operative findings are described in detail below for 5 exemplar patients. The obstruction occurred due to either a thickened arachnoid "veil" or scarring, or obliteration by a cerebellar tonsil. A veil was most common and was found in 9 patients with covering of the outlet of the fourth ventricle ${ }^{6}$ (foramen of Magendie). Either the right or left or both tonsils blocked the egress out of the fourth ventricle in 4 patients. In 3 of these individuals, a tonsil filled the entire fourth ventricle outlet.

Intraoperative observation revealed the opening of the 


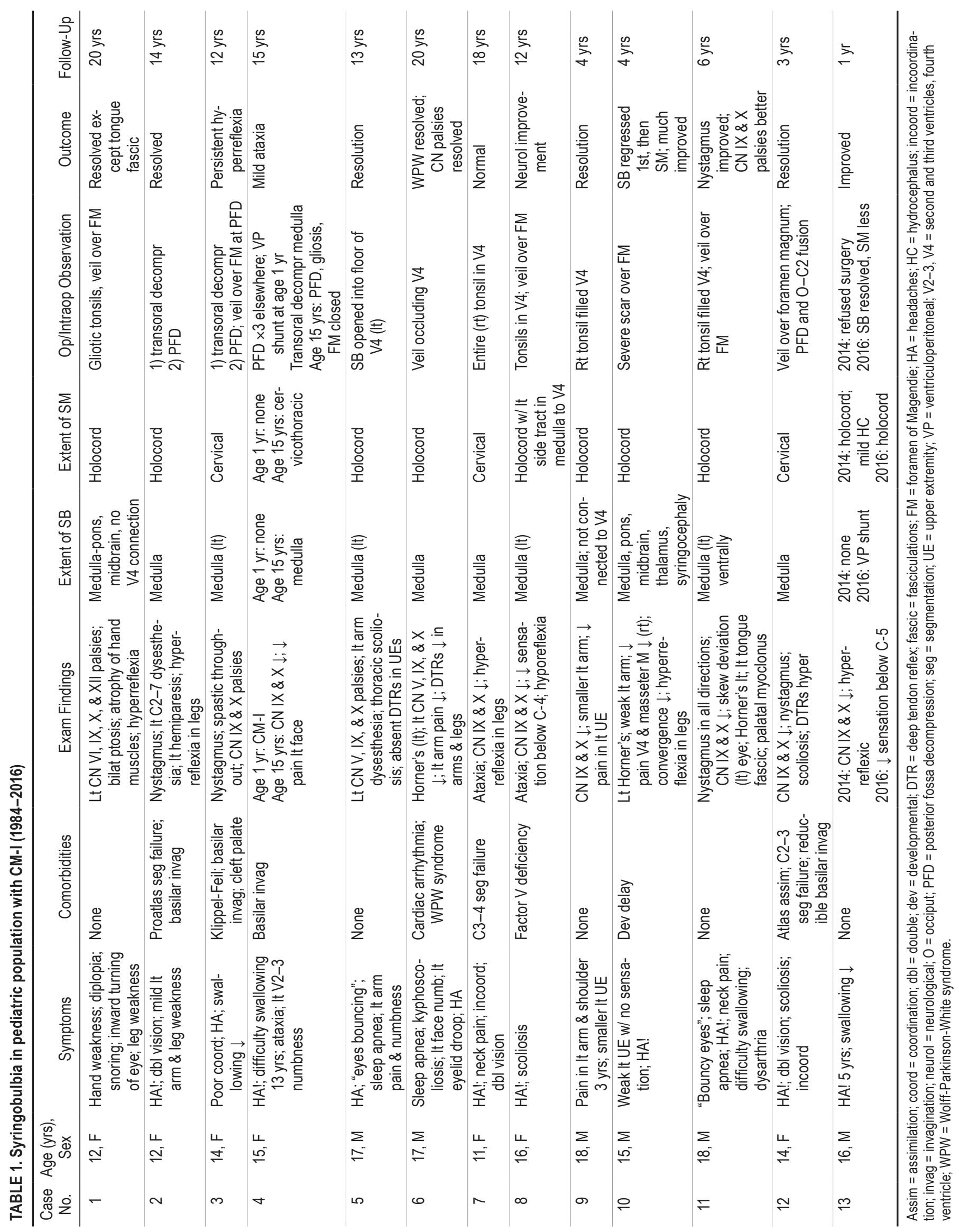



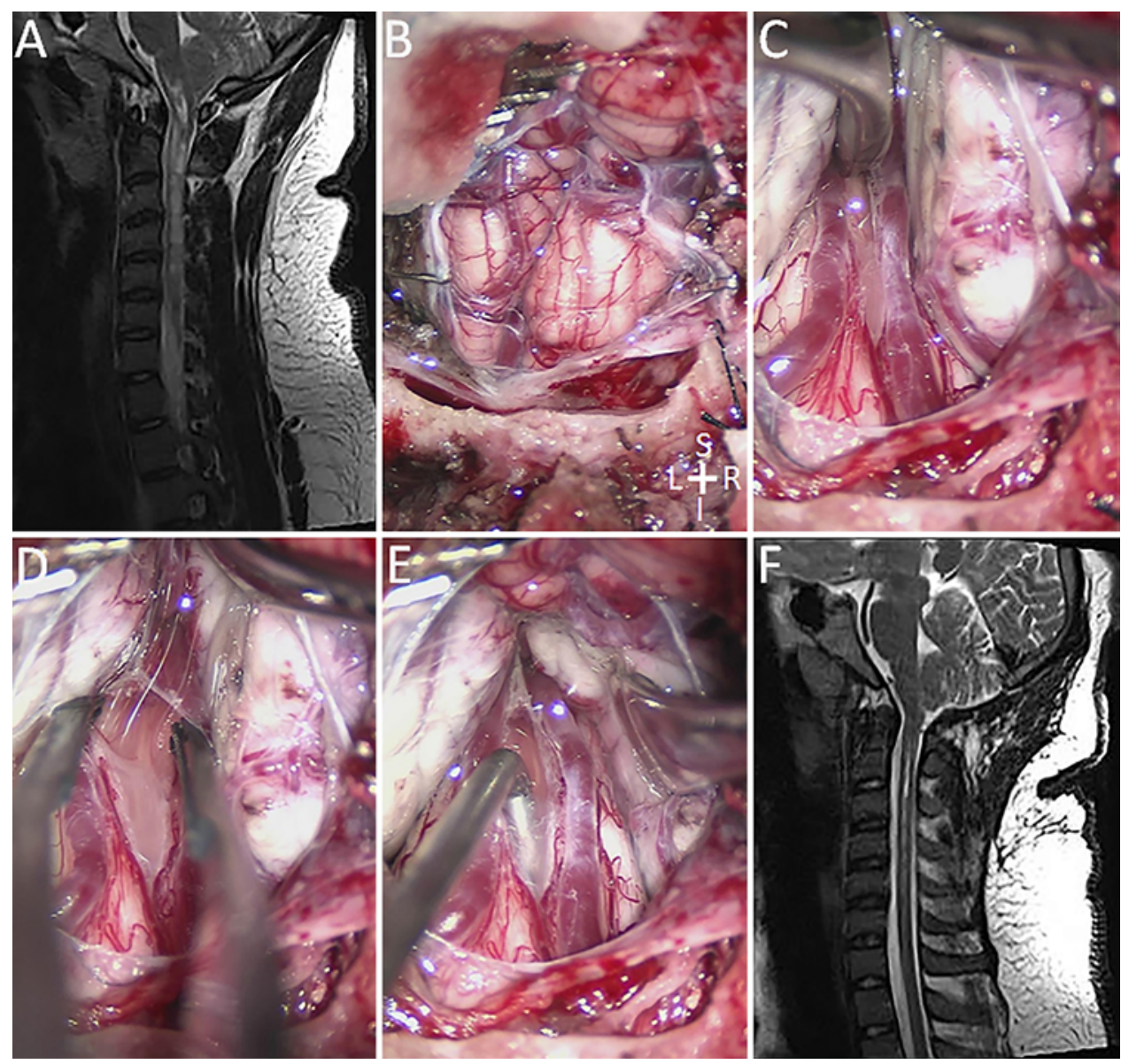

FIG. 1. Case 11. A: Preoperative midsagittal T2-weighted MR image of CVJ. Note tonsillar ectopia, SM, and SB in medulla. B: Intradural photograph obtained after posterior fossa decompression with partial C-1 laminectomy. The cerebellar tonsils are medially approximated and overlie the FM. C: The vermian vessels are over the roof of the fourth ventricle. There is an arachnoid veil over the foramen of Magendie. D: Arachnoid veil over the foramen of Magendie exposed. E: Opening of arachnoid veil. F: Postoperative MR image showing resolution of the SB and SM. In all photographs, L (left), R (right), S (superior), and I (inferior) denote orientation. All MR images utilize standard radiological orientation.

$\mathrm{SB}$ in the floor of the fourth ventricle in one patient as demonstrated (Case 5). The preoperative MRI revealed communication of the SB with the fourth ventricle directly via a lateral cleft. In 6 patients there was no communication with the fourth ventricle.

\section{Outcomes}

The SB improved in all cases. The SM resolved in 12 of the 13 patients. In the 1 patient with syringocephaly (Case 10), the syringocephaly and the SB were the first to resolve (within 6 weeks), followed by resolution of the SM. Cranial nerve palsies resolved, except for CN XII palsies in 2 patients; these patients had tongue fasciculations present preoperatively and these persisted postoperatively.

\section{Representative Cases \\ Case 11}

This 18-year-old male presented with acute onset of trouble focusing, especially with far distance and when driving. He complained of double vision when looking to the right. Headaches and neck pain prompted chiroprac- tic manipulations. His speech became slurred, and he also had a history of sleep apnea. On examination he was moderately obese. He had nystagmus in all directions of gaze and downbeat nystagmus. There was outward deviation of the right eye. He had dysarthria, fasciculations in the left side of his tongue, and palatal myoclonus. His gait was unsteady. MRI revealed CM-I with the tip of the ectopic cerebellar tonsil reaching the upper border of the posterior atlas arch (Fig. 1A). The cervicomedullary buckle was at this level. There was holocord SM with a medullary SB that was located to the left of the midline. The inferior portion of this connected with the SM.

At operation an intradural procedure demonstrated the cerebellar tonsils to be medially approximated and extending down to the posterior arch of C-1 (Fig. 1B). The vermian branches of the posterior inferior cerebellar artery were overriding the exit of the fourth ventricle (Fig. 1C). An arachnoid veil was present at the inferior third of the foramen of Magendie (Fig. 1D and E) and was excised.

Postoperative MRI showed resolution of the SB and marked reduction of the SM (Fig. 1F). At 6-year followup, the nystagmus had nearly resolved except for partial 

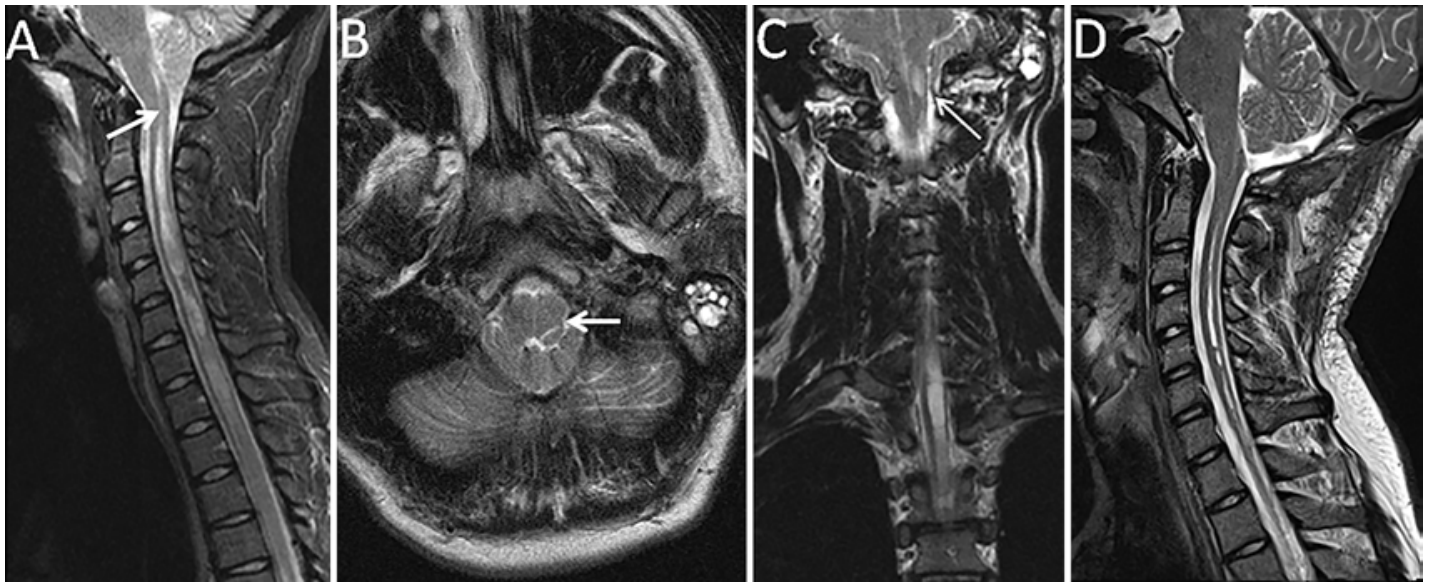

FIG. 2. Case 9. A: Preoperative midsagittal T2-weighted MR image of CVJ. Note the SM with extension into the medulla (arrow). B: Axial T2-weighted MR image through foramen magnum. There is tonsillar impaction and a slit SB in the medulla to the left of midline (arrow). C: Coronal T2-weighted MR image with medullary SB visualized to the left of midline (arrow). D: Postoperative midsagittal T2-weighted MR image showing that SM and SB have regressed. All MR images utilize standard radiological orientation.

downbeat nystagmus. Tongue fasciculations still persisted. The patient's sleep apnea and ataxia had resolved.

\section{Case 9}

This 18-year-old male presented with a 2-year history of pain as well as paresthesias in his left upper extremity, which he felt was weakening. He had received chiropractic attention over 2 years. He claimed to have had complete loss of sensation in his left arm and leg for a year. On examination, his head was cocked to the right. He had a partial cranial nerve $(\mathrm{CN})$ VI palsy. The gag response was absent bilaterally. His left triceps was extremely weak, and he had atrophy of the muscles of the medial forearm of his left hand. His grip strength was weak bilaterally but worse in the left hand than the right hand. He had good strength in the lower extremities. Light touch was interpreted as pain in the left arm and left leg. The deep tendon reflexes were absent in the upper extremities and were grossly hyperreflexic in the lower extremities. His station and gait were normal. MRI revealed cerebellar tonsillar ectopia (Fig. 2A) with impaction at the foramen magnum on axial views (Fig. 2B). There was a left medullary SB (Fig. 2A-C) and holocord SM (Fig. 2A and C).

At posterior fossa exploration, the right tonsil bulged into the fourth ventricle. Inspection of the floor of the fourth ventricle failed to identify any visualization of the medullary syrinx. His postoperative evaluation 4 years later showed that he was doing well and gainfully employed. His gag response was normal. The extraocular movements were full and he was strong throughout. Sensation to touch and pinprick had returned to normal. The deep tendon reflexes, however, were hypoactive. Postoperative MRI showed resolution of the SB and marked reduction of the SM (Fig. 2D).

\section{Case 5}

This 17-year-old male presented with scoliosis and complaints of "bouncing of my eyes." He had occipital headaches which were worsened with Valsalva. He also had sleep apnea. He complained of a painful sensation in his left arm. On examination, he had cervicothoracic kyphoscoliosis, weakness of his left masseter muscle, numbness in the left face, and CN IX and X palsies. He also had dysesthesia in his left arm. The deep tendon reflexes were absent in the upper extremities.

MRI revealed CM-I with holocord SM (Fig. 3A and B) and left-lateralized medullary SB (Fig. 3A-C). Parasagittal MRI suggested communication of the SB into the floor of the fourth ventricle (Fig. 3A and B). At posterior fossa decompression, the cerebellar tonsils were herniated below the posterior arch of C-1, and the left cerebellar tonsil was gliotic (Fig. 3D). The foramen of Magendie was covered with an arachnoid scar (Fig. 3E). Once this was opened, we recognized a pinkish-colored opening in the floor of the fourth ventricle to the left of the midline (Fig. $3 \mathrm{~F}$ and $\mathrm{G})$. This was the communication of the SB into the fourth ventricle. At follow-up, the patient had resolution of his symptoms and his postoperative follow-up MRI showed regression of the SB and SM (Fig. 3H). It has now been 13 years since his surgery.

\section{Case 1}

This 12-year-old girl presented in 1995 with relatively sudden onset of weakness in her hands and double vision. On detailed questioning she had snoring and complained of weakness in her left leg. On examination she had bilateral ptosis, and left CN VI, IX, and X palsies with atrophy of the tongue. She had atrophy of both hands and hyperreflexia. MRI demonstrated CM-I with SB in the medulla and pons and into the midbrain (Fig. 4A-C). There was no suggestion of communication with the fourth ventricle on MRI. She had a holocord SM.

At posterior fossa decompression, bilateral gliotic cerebellar tonsils were visualized (Fig. 4C) and a veil over foramen of Magendie (Fig. 4D and E) was identified and opened. Her symptoms resolved postoperatively, except for the tongue fasciculations. It has now been 20 years since her surgical intervention. 

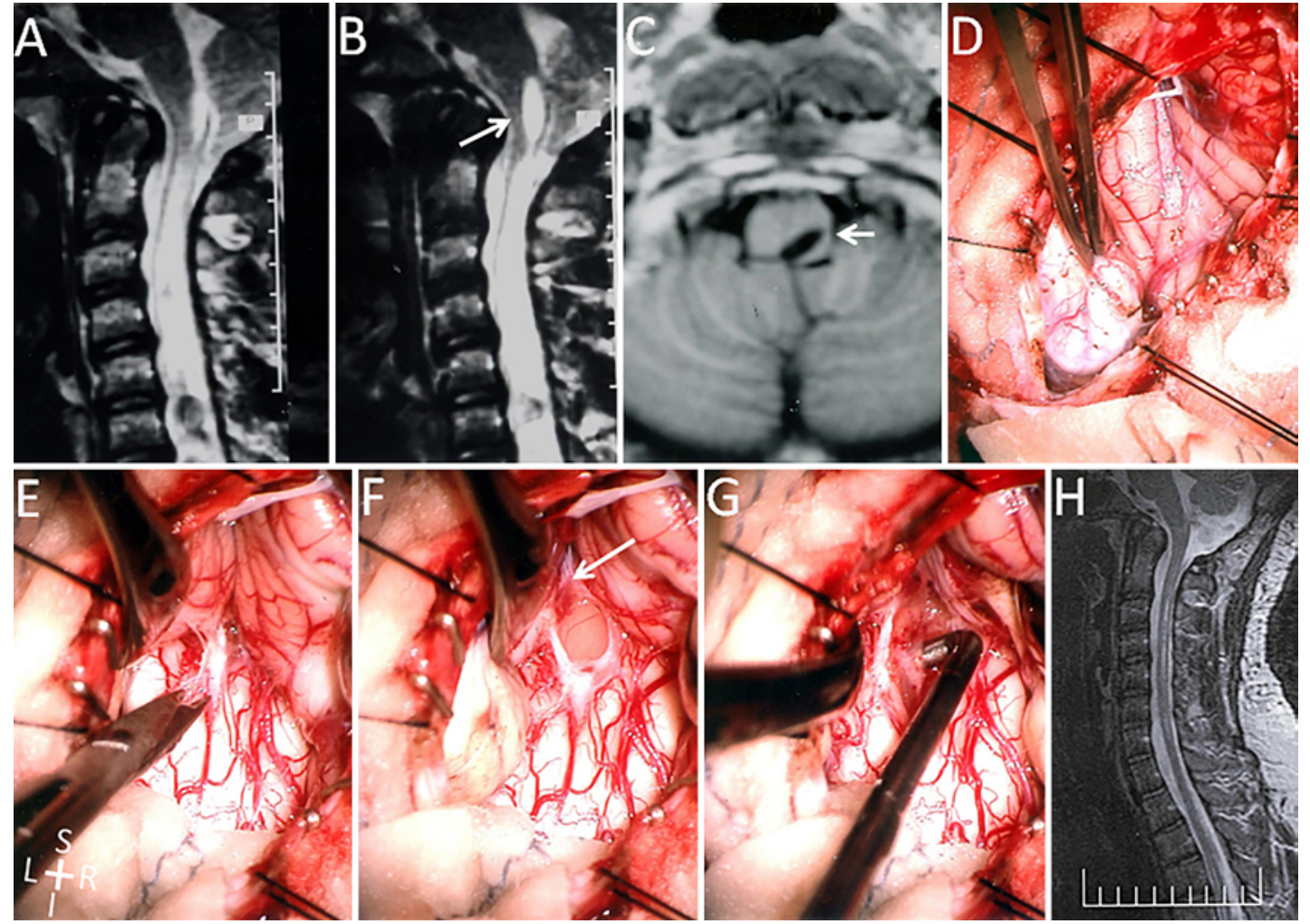

FIG. 3. Case 5. A: Preoperative midsagittal T2-weighted MR image of CVJ showing SM and medullary SB. B and C: Parasagittal T2-weighted (B) and axial T1-weighted (C) images showing SB location left of midline in the medulla (arrows). D: Operative photograph showing gliotic left cerebellar tonsil being elevated above the $\mathrm{C}-1$ arch. E: Arachnoid being opened over the foramen of Magendie. F: A pink discoloration was noted in the floor of the fourth ventricle (arrow), which was found to be an opening and communication into the SB. G: Probe in connection of the fourth ventricle to SB. H: Postoperative midsagittal T2-weighted MR image demonstrating resolution of the SM and SB. In all photographs, L (left), R (right), S (superior), and I (inferior) denote orientation. All MR images utilize standard radiological orientation.

\section{Case 7}

This 11-year-old girl presented with headaches, neck pain, and incoordination of her hands in 1999. She also complained of double vision. Her comorbidities included segmentation failure of C-3 and C-4. On examination she had truncal ataxia, hyperreflexia in her lower extremities, and CN IX and X palsies. MRI showed that SM was present in the cervical region with a lateral cleft in the medulla coming off the fourth ventricle (white arrow, Fig. 5A) with the cerebellar tonsil filling the fourth ventricle (red arrow, Fig. 5A). This was confirmed at posterior fossa decompression (Fig. 5B-D). Postoperatively she had recovery of function, and it is now 18 years since her operation.

\section{Discussion}

SB refers to a longitudinally oriented fluid-filled cavity within the brainstem, intimately associated with SM. . $^{36,38}$ SB can accompany varied pathologies, including CM-I, CM-II, inflammatory disorders such as arachnoiditis and meningitis, trauma, and tumors of the posterior fossa as well as the spinal cord. Our series of 13 pediatric patients with SB and CM-I demonstrates clear patterns.

First, the presentation of SB is usually chronic. Nevertheless, acute presentations have been reported..$^{12}$ In 2011, Massey et al. reported on a 4-year-old patient with acute
CN VI palsy along with other cranial nerve abnormalities. ${ }^{16}$ Acute hoarseness of voice with sudden onset of CN X palsy has been described on several occasions. $7,10,11,14,23,32,43$ In our series, acute onset of neurological deficit occurred in 2 patients with violent coughing, and subacute onset was seen in 3 .

Headaches are the most common presenting symptom of CM-I. CM-I-associated SM can result in spinal cord symptoms and scoliosis. However, cranial nerve dysfunction associated with CM-I has been poorly recognized. CM-I-associated SB invariably results in cranial nerve dysfunction. The finding of an absent gag response in 12 of our 13 patients is important, as CN IX and CN X were the cranial nerves most commonly affected. Trigeminal dysfunction was seen in 5 patients. This has been reported as difficulty with jaw opening or mastication and the presence of facial pain. ${ }^{18,20,23,26,28,29}$ Convergence difficulties and Horner's syndrome were present in 5 other patients. Whether this is part of the SM or the SB is difficult to ascertain. However, in 1 patient with syringocephaly whose case we previously described, the ophthalmological findings of internuclear ophthalmoplegia came on suddenly. ${ }^{17}$

Nogués et al. reviewed the ophthalmological complications of SB with CM-I. ${ }^{23,24}$ They described 22 patients with SB and found a trigeminal sensory loss in 5. In their experience, SB appeared in 3 different positions. The first was 

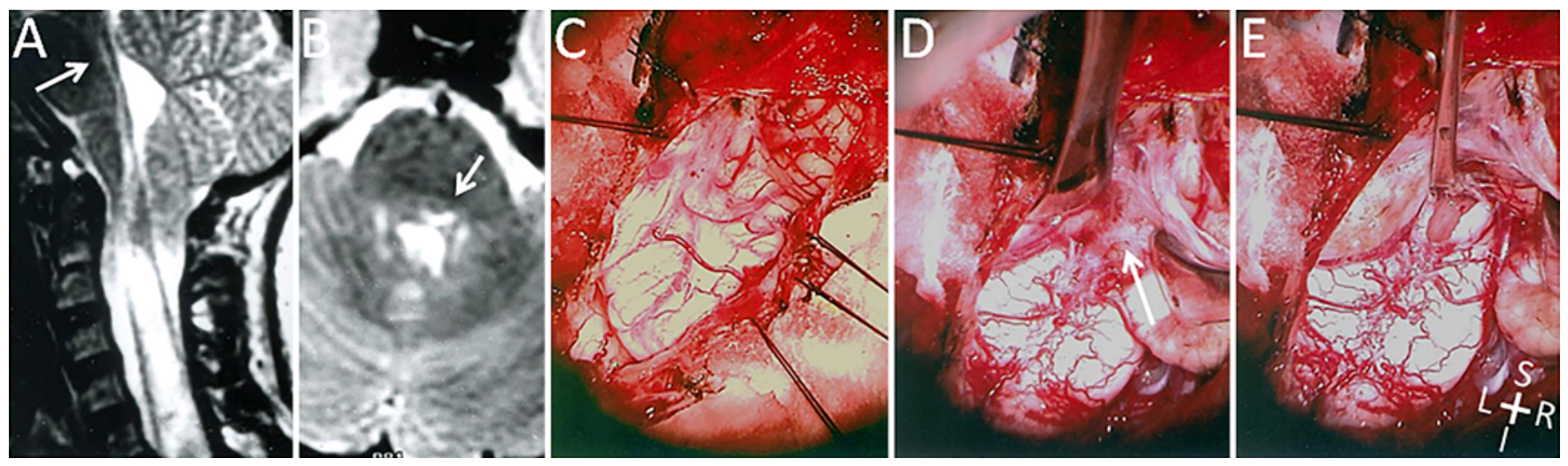

FIG. 4. Case 1. A: Midsagittal T2-weighted MR image showing SM and SB involving the medulla and pons (arrow). B: Axial T2-weighted image through the pons showing SB distinct from the fourth ventricle and eccentric to the left (arrow). C: The cerebellar tonsils extend below the C-1 arch and overlie the foramen of Magendie. D: Adhesions occlude the foramen of Magendie (arrow). E: Exposure of the floor of the fourth ventricle after incision of occluding scar. In all photographs, L (left), R (right), S (superior), and I (inferior) denote orientation. All MR images utilize standard radiological orientation.

from the floor of the fourth ventricle and was anterolateral. A second location was along the midline floor of the fourth ventricle, and these cases were the least symptomatic. The last was a medial location, ventral to the fourth ventricle. Some of these cases were in the MRI era and were documented with both sagittal and axial views. The authors described acute symptoms of SB as involving vertigo, sudden onset of oscillopsia, nausea, and ataxia. The insidious onset was associated with hoarseness of voice and dysphagia. They felt that vestibular and trigeminal symptoms were characteristic of SB. Horner's syndrome was present in $18 \% .{ }^{39}$ Williams and Morgan reviewed their experience of SB with similar findings. ${ }^{21,45}$ Similarly Valentini and colleagues defined the SB cavities in their experience with CM-I. ${ }^{41,42}$

Cardiovascular and autonomic responses to SM and SB have been reported as rhythm abnormalities as seen in our Case 6. ${ }^{10,23,37}$ Horner's syndrome has been described with SM as well as SB. . $^{10,13,17,23,27}$ Giant SB in childhood and fatal hemorrhage have been reported. ${ }^{31,34,35}$ Intractable hiccups $^{4,33}$ have been observed in SB and SM, and in 1 case the hiccups resolved after posterior fossa surgery. ${ }^{33}$
Another consistent observation we report relates to SB location: SB in the medulla was present in all of our 13 cases. In only 2 cases did it extend further rostrally into the pons and midbrain and/or cerebrum. In several cases the SB was eccentrically located within the medulla, and the SB was felt to communicate with the fourth ventricle in 7 of the 13 cases.

Our series demonstrates the close link between SB and SM-every patient with SB had SM. All patients had SM involving the cervical cord, and in all but 1 case the SM cavities were very large, involving the entire cord. We postulate that SB arises due to upward extension of the SM into the fourth ventricle consistent with the "slosh" mechanism described by Williams during the Valsalva maneuver. ${ }^{44}$ However, the eccentric SB probably represents a disruption in the central canal at the level of the SM to allow for ascent along the fiber tracts. The fact that the SB and SM improved dramatically after posterior fossa decompression points to the pathology being at the egress of the fourth ventricle with impaction of the tonsils or the intradural pathology and relief with decompression. ${ }^{6}$

Indeed, some form of fourth ventricle egress obstruc-
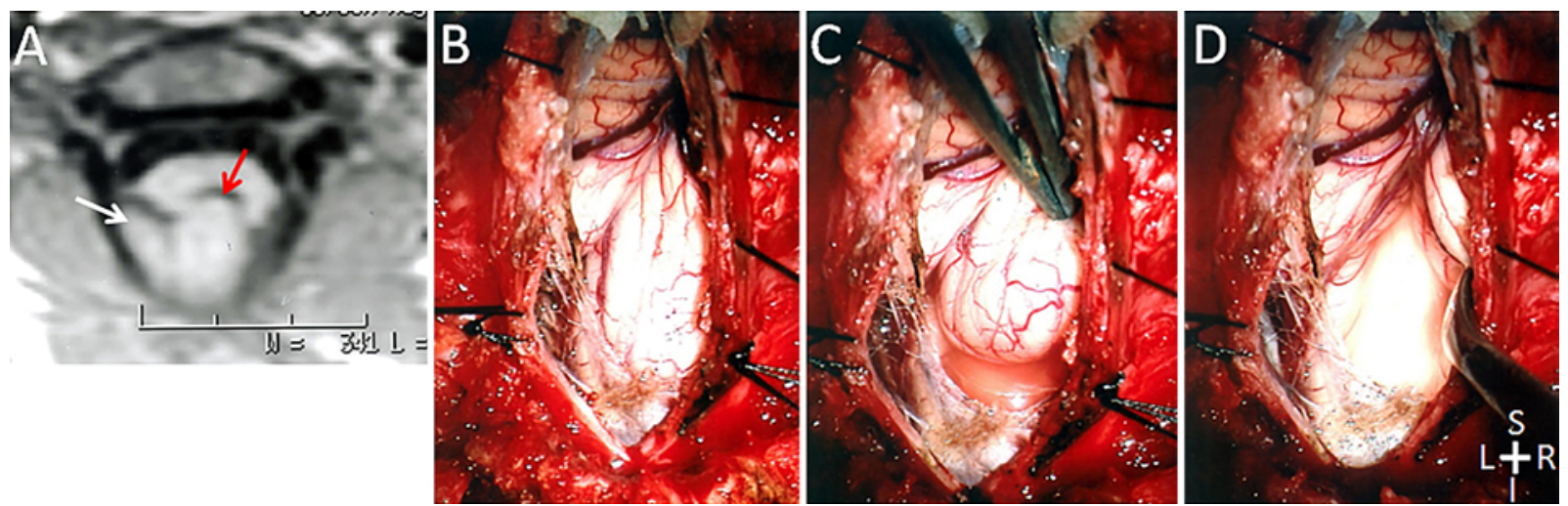

FIG. 5. Case 7. A: Axial T1-weighted MR image through the medulla. Note the right tonsil in the fourth ventricle (red arrow) and lateral cleft off the fourth ventricle (white arrow). B: Operative view of cerebellum and dorsal cervicomedullary junction. The right tonsil extends to C-2. C: The right cerebellar tonsil fills the fourth ventricle. D: Fourth ventricle floor exposed after right tonsil is shrunk. In all photographs, L (left), R (right), S (superior), and I (inferior) denote orientation. All MR images utilize standard radiological orientation. 
tion was identified at surgery in all patients at the time of surgery. Most commonly, an arachnoid veil was seen occluding the foramen of Magendie; this was documented in 9 of the 13 patients. A tonsil was seen to occlude the fourth ventricle in some patients. Significant scarring between the cerebellar tonsils was seen in 3 of 13. The arachnoid abnormalities we found raise the question of whether previous infection or subarachnoid hemorrhage at the time of birth contribute to eventual development of SM. ${ }^{17}$ Hydrocephalus was seen in only 1 patient (Case 13), but initial treatment with ventriculoperitoneal shunting proved unsuccessful in resolving the SM and SB, and the patient ultimately required a posterior fossa procedure a year later.

Follow-up clinical evaluation and MRI in our patients showed that the SB improved before SM. The cranial nerve palsies resolved in 11 of the 13. Neurological improvement was invariable.

\section{Conclusions}

Pediatric patients with CM-I and SM are at risk for developing SB. With the pathology described at the fourth ventricle outlet, an intradural procedure is recommended, with lysis of adhesions and careful inspection of the floor of the fourth ventricle. Establishing egress out of the fourth ventricle has been the goal, with or without obvious communication with the SB to the fourth ventricle, and we believe that a duraplasty is a critical component of the procedure. Our database and archive retrieval has helped in establishing the pathology and management of this rare entity, showing that this surgical methodology is effective in treating SB.

\section{References}

1. Abe K, Sawada H, Fujiwara M, Mezaki T, Udaka F, Kitahara Y, et al: MR imaging of syringobulbia with giant syrinx. Neuroradiology 30:442-443, 1988

2. Afifi AK, Menezes AH: Rostral slit-like extension of holochord syringomyelic cavity: childhood-onset case and review of literature. J Child Neurol 12:515-518, 1997

3. Aryan HE, Yanni DS, Nakaji P, Jandial R, Marshall LF, Taylor WR: Syringocephaly. J Clin Neurosci 11:421-423, 2004

4. Batzdorf U: Treatment of syringomyelia associated with Chiari I malformation, in Tamaki N, Batzdorf U, Nagashima T (eds): Syringomyelia. Current Concepts in Pathogenesis and Management. Tokyo: Springer, 2001, pp 121-135

5. Del Bigio MR, Deck JHN, MacDonald JK: Syrinx extending from conus medullaris to basal ganglia: a clinical, radiological, and pathological correlation. Can J Neurol Sci 20:240246, 1993

6. Dlouhy BJ, Dawson JD, Menezes AH: Intradural pathology and pathophysiology associated with Chiari I malformation in children and adults with and without syringomyelia. J Neurosurg Pediatr 20:526-541, 2017

7. Gennaro P, Miller M: Syringobulbia without syringomyelia. Arch Neurol 40:394, 1983 (Letter)

8. Gilbert JN, Jones KL, Rorke LB, Chernoff GF, James HE: Central nervous system anomalies associated with meningomyelocele, hydrocephalus, and the Arnold-Chiari malformation: reappraisal of theories regarding the pathogenesis of posterior neural tube closure defects. Neurosurgery 18:559_ 564,1986

9. Greenlee JDW, Menezes AH, Bertoglio BA, Donovan KA: Syringobulbia in a pediatric population. Neurosurgery 57:1147-1153, 2005
10. Heidel KM, Benarroch EE, Gené R, Klein F, Meli F, Saadia $\mathrm{D}$, et al: Cardiovascular and respiratory consequences of bilateral involvement of the medullary intermediate reticular formation in syringobulbia. Clin Auton Res 12:450-456, 2002

11. Jha S, Das A, Gupta S, Banerji D: Syringomyelia with syringobulbia presenting only with paralysis of 9th and 10th cranial nerves. Acta Neurol Scand 105:341-343, 2002

12. Kanev PM, Getch CC, Jallo J, Faerber EN: Cerebral syrinx with Chiari I malformation. Pediatr Neurosurg 20:214-216, 1994

13. Kerrison JB, Biousse V, Newman NJ: Isolated Horner's syndrome and syringomyelia. J Neurol Neurosurg Psychiatry 69:131-132, 2000

14. Kombos T, Ramsbacher J, Brock M: [Isolated idiopathic syringobulbia: case report and summary of the literature.] Zentralbl Neurochir 57:103-107, 1996 (Ger)

15. Lee JH, Chung CK, Kim HJ: Cerebral dissection from syringomyelia demonstrated using cine magnetic resonance imaging. Case report. J Neurosurg 94:318-321, 2001

16. Massey SL, Buland J, Hauber S, Piatt J Jr, Goraya J, Faerber $\mathrm{E}$, et al: Acute VI nerve palsy in a 4 year-old girl with Chiari I malformation and pontomedullary extension of syringomyelia: case report and review of the literature. Eur J Paediatr Neurol 15:303-309, 2011

17. Menezes AH, Greenlee JDW, Longmuir RA, Hansen DR, Abode-Iyamah K: Syringohydromyelia in association with syringobulbia and syringocephaly: case report. J Neurosurg Pediatr 15:657-661, 2015

18. Merello M, Lees AJ, Leiguarda R, Guevara J, Nogués M: Inverse masticatory muscle activity due to syringobulbia. Mov Disord 8:359-360, 1993

19. Milhorat TH, Capocelli AL Jr, Anzil AP, Kotzen RM, Milhorat RH: Pathological basis of spinal cord cavitation in syringomyelia: analysis of 105 autopsy cases. J Neurosurg 82:802-812, 1995

20. Mitani K, Toyooka K, Kaido M, Yuasa R, Hamada S, Tachimura T: [Disturbance of jaw-opening due to extension of syringobulbia to the pons-a case report.] Rinsho Shinkeigaku 42:540-543, 2002 (Jpn)

21. Morgan D, Williams B: Syringobulbia: a surgical appraisal. J Neurol Neurosurg Psychiatry 55:1132-1141, 1992

22. Moriwaka F, Tashiro K, Tachibana S, Yada K: [Epidemiology of syringomyelia in Japan - the nationwide survey.] Rinsho Shinkeigaku 35:1395-1397, 1995 (Jpn)

23. Nogués M, López L, Meli F: Neuro-ophthalmologic complications of syringobulbia. Curr Neurol Neurosci Rep 10:459-466, 2010

24. Nogués MA, Benarroch E: Abnormalities of respiratory control and the respiratory motor unit. Neurologist 14:273-288, 2008

25. Okada S, Nakagawa Y, Hirakawa K: Syringomyelia extending to the basal ganglia. Case report. J Neurosurg 71:616617, 1989

26. Peñarrocha M, Okeson JP, Peñarrocha MS, Angeles Cervelló M: Orofacial pain as the sole manifestation of syringobulbiasyringomyelia associated with Arnold-Chiari malformation. J Orofac Pain 15:170-173, 2001

27. Pomeranz $\mathrm{H}$ : Isolated Horner syndrome and syrinx of the cervical spinal cord. Am J Ophthalmol 133:702-704, 2002

28. Priyadarshini H, Harish A: Syringomyelia with syringobulbia. J Assoc Physicians India 55:855, 2007

29. Riaz G, Campbell WW, Carr J, Ghatak N: Facial myokymia in syringobulbia. Arch Neurol 47:472-474, 1990

30. Robinson L, Bisnaire D: Syringomyelia and syringobulbia: pathophysiology, surgical treatment and nursing implications. J Neurosci Nurs 22:69-75, 1990

31. Roig C, Lopez-Pousa S, Ferrer I: Bleeding in syringobulbia. A fatal complication. Eur Neurol 21:189-193, 1982

32. Romero-Pinel L, Fernández S, Torres A, Majós C, Ferran E, 
Martínez-Yélamos S, et al: Syringomyelia extending to the corona radiata. J Neurol 253:817-818, 2006

33. Seki T, Hida K, Lee J, Iwasaki Y: Hiccups attributable to syringobulbia and/or syringomyelia associated with a Chiari I malformation: case report. Neurosurgery 54:224-227, 2004

34. Sengar RLS, Prasad VSSV, Prasad BCM, Reddy DR: Giant syringobulbia in childhood. Childs Nerv Syst 10:350-351, 1994

35. Shuster A, Landry D: Case 191: giant hemorrhagic syringomyelia and syringobulbia mimicking intramedullary neoplasm. Radiology 266:991-993, 2013

36. Spiller WG: Syringomyelia, extending from the sacral region of the spinal cord through the medulla oblongata, right side of the pons and right cerebral peduncle to the upper part of the right internal capsule (syringobulbia). BMJ 2:1017-1021, 1906

37. Stovner LJ, Kruszewski P, Shen JM: Sinus arrhythmia and pupil size in Chiari I malformation: evidence of autonomic dysfunction. Funct Neurol 8:251-257, 1993

38. Taghipour M, Derakhshan N, Ghaffarpasand F: Isolated posttraumatic syringobulbia; case report and review of the literature. Bull Emerg Trauma 2:166-169, 2014

39. Trobe JD: The evaluation of Horner syndrome. J Neuroophthalmol 30:1-2, 2010

40. Tubbs RS, Bailey M, Barrow WC, Loukas M, Shoja MM, Oakes WJ: Morphometric analysis of the craniocervical juncture in children with Chiari I malformation and concomitant syringobulbia. Childs Nerv Syst 25:689-692, 2009

41. Valentini MC, Bracchi M, Gaidolfi E, Savoiardo M: Radiologic demonstration of syringobulbia. Report of 8 cases. Acta Radiol Suppl 369:245-247, 1986
42. Valentini MC, Forni C, Bracchi M: Syringobulbia extending to the basal ganglia. AJNR Am J Neuroradiol 9:205-207, 1988

43. Viswanatha B: Syringomyelia with syringobulbia presenting as vocal fold paralysis. Ear Nose Throat J 88:E20, 2009

44. Williams B: Simultaneous cerebral and spinal fluid pressure recordings. 2. Cerebrospinal dissociation with lesions at the foramen magnum. Acta Neurochir (Wien) 59:123-142, 1981

45. Williams B: Syringobulbia: a surgical review. Acta Neurochir (Wien) 123:190-194, 1993

\section{Disclosures}

The authors report no conflict of interest concerning the materials or methods used in this study or the findings specified in this paper.

\section{Author Contributions}

Conception and design: Menezes. Acquisition of data: all authors. Analysis and interpretation of data: Menezes, Greenlee. Drafting the article: Menezes. Critically revising the article: all authors. Reviewed submitted version of manuscript: all authors. Approved the final version of the manuscript on behalf of all authors: Menezes. Statistical analysis: Menezes.

\section{Correspondence}

Arnold H. Menezes: University of Iowa Stead Family Children's Hospital, Iowa City, IA. arnold-menezes@uiowa.edu. 